\title{
l. Nuevos espacios para la construcción de la Iglesia: Estado nacional y sectores ultramontanos en la Confederación Argentina, 1853-1862
}

Ignacio Martínez ${ }^{1}$

\begin{abstract}
Resumen
En este artículo se analizan dos aspectos centrales de la construcción eclesiástica en la Confederación Argentina durante la década de 1850: el financiamiento del culto por parte del Estado y el proceso de provisión de sus tres diócesis. Se intenta demostrar que, en esa construcción, Estado y Santa Sede funcionaron más como espacios de confluencia de actores concretos, que como actores mismos. Se vincula esta hipótesis con nuevos enfoques sobre la formación del Estado nacional y sobre la romanización.
\end{abstract}

Palabras clave: Argentina; siglo XIX; Iglesia Católica; Santa Sede; Estado nacional.

New spaces for the construction of the Church: national state and ultramontane sectors in Argentine Confederation, 1853-1862

\begin{abstract}
Two aspects of the building of Argentinian Church in the 1850s are studied: the state funding for Catholic Church and the appointment of bishops for the three Argentinian dioceses. The aim of this article is to prove that the State and the Holy See worked as places in this process where political and ecclesiastical agents pursued their own goals. This hypothesis is linked with new approaches on Nation-state building and romanization.
\end{abstract}

1 Universidad Nacional de Rosario/Consejo Nacional de Investigaciones Científicas y Tecnológicas. Argentina. Correo electrónico: igntinez@gmail.com 
Key words: Argentina; 19th Century; Catholic Church; Holy See; Nation-state.

Fecha de recepción de originales: 11/11/2014.

Fecha de aceptación para publicación: 03/07/2015.

\section{Nuevos espacios para la construcción de la Iglesia: Estado nacional y sectores ultramontanos en la Confederación Argentina, 1853-1862}

\section{Introducción}

a experiencia política iniciada por las trece provincias que se reunieron en — Santa Fe en 1853 para sancionar una Constitución nacional está siendo revisada últimamente con el objetivo de discutir interpretaciones previas sobre el proceso más amplio de construcción del Estado en la Argentina. Las imágenes cuestionadas son, fundamentalmente, dos. Primero, que durante ese proceso las élites provinciales asistieron de forma más o menos pasiva a la reducción, por parte del Estado central, de los espacios y herramientas de poder que ejercían a escala local desde 1820. Segundo, que esa centralización fue posible recién cuando el Estado nacional contó con los recursos económicos de la aduana de Buenos Aires para poner en marcha diferentes estrategias de "penetración" del poder central en los espacios provinciales. Desde esta perspectiva, el gobierno que encabezaba Justo José de Urquiza se caracterizó más por las intenciones frustradas que por logros efectivos, en razón de que no había contado con los recursos materiales suficientes para torcer el brazo a los poderes locales. ${ }^{2}$ Las interpretaciones recientes matizan, precisamente, esos dos supuestos. En primer lugar, se destaca la participación activa de las élites provinciales en la construcción de un poder nacional institucionalizado, puesto que allí encontraron la oportunidad de incrementar, más que de reducir, sus ámbitos de influencia. En segundo, y lógicamente vinculado a esta tesis, el foco del análisis se concentra en instituciones y espacios políticos que no requerían necesariamente de grandes erogaciones económicas para prosperar, de allí que los esfuerzos nacionalizadores durante la secesión de Buenos Aires se presenten menos estériles que en perspectivas anteriores (Bragoni y Míguez, 2010; Míguez, 2012). ${ }^{3}$

\footnotetext{
2 La mejor sistematización de esta interpretación es la del clásico libro de Oszlak (1985). Considero, de todas maneras, que la tipología que traza para pensar las formas de "penetración" del Estado nacional en el territorio que desea controlar puede ser orientativa, siempre que se despoje al concepto del rol pasivo que otorga a los gobiernos y élites provinciales.
}

3 Así ocurrió, por ejemplo, durante la puesta en marcha del Congreso nacional en 1854 (Lanteri, 2013a). 
El espacio eclesiástico es uno de los ámbitos donde el gobierno nacional de la década de 1850 intervino y consiguió cumplir relativamente bien sus objetivos. Durante este período se recabaron datos sobre la situación de las iglesias de todas las provincias, se enviaron misiones diplomáticas a la Santa Sede, se proveyeron todas las diócesis vacantes, se creó el obispado del Litoral y, finalmente, se recibió un emisario de Roma con carácter de delegado apostólico, lo que se consideraba el paso previo a la instalación de una nunciatura. Sin embargo, fueron pocos los investigadores que vincularon esta política con el proceso de creación del Estado nacional siguiendo la renovación que acabamos de mencionar. ${ }^{4}$ La inclusión de la dimensión eclesiástica como parte de la construcción estatal vino del lado de los estudiosos de la historia de la Iglesia. La hipótesis disparadora fue planteada por Roberto Di Stefano (2004; Di Stefano y Zanatta, 2000). Su interpretación abarca un período más largo: sostiene que la formación del Estado y la Iglesia contemporáneos en Argentina fueron procesos paralelos y, en cierto grado, interdependientes. Según el autor, esa dinámica comenzó a nivel provincial en Buenos Aires durante las reformas rivadavianas de la década de 1820 y cobró dimensión nacional en el período que va de la Constitución de 1853 a la elevación de la sede diocesana porteña a metropolitana en 1865. Sobre esta línea, Miranda Lida publicó dos trabajos que hacen hincapié en el protagonismo del gobierno de la Confederación en la construcción de la Iglesia argentina. En primer lugar, porque fue una fuente importante de recursos materiales (Lida, 2007), y en segundo, porque garantizó el marco legal y político necesario para el fortalecimiento institucional de la Iglesia (Lida, 2006). Aunque ambos autores parten de la misma premisa, difieren en sus hipótesis a la hora de evaluar el peso relativo de los actores involucrados. Di Stefano considera que el surgimiento de la Iglesia contemporánea es producto de la acción coincidente del Estado argentino en su búsqueda por centralizar la institución eclesiástica como parte del proceso de concentración de la soberanía, y de la Santa Sede, embarcada en un proceso de centralización del poder conocido como "romanización". Lida (2006), por su parte, sostiene que solo el primero fue una variable determinante de la construcción eclesiástica argentina entre los años 1852 y 1865 . Su posición se sustenta en un cuestionamiento del concepto de "romanización", que entiende como un proceso dirigido unilateralmente desde Roma sobre las iglesias periféricas o nacionales. La autora considera que la influencia romana actuó sobre la estructura eclesiástica argentina solo cuando el poder político se lo permitió. Por el contrario,

\footnotetext{
4 Es sintomático que la política eclesiástica ni siquiera se mencione en recientes obras de síntesis sobre el período, en balances historiográficos o en obras colectivas que buscan reflejar los nuevos enfoques. Ver respectivamente: Bragoni y Míguez (2010), Sábato (2012), Míguez (2012).
} 
cuando la autoridad papal dejó de ser funcional a la formación de la Iglesia "a la medida del Estado", el gobierno obstaculizó la labor del delegado pontificio hasta provocar su salida del país.

Este artículo busca terciar entre estas posiciones modificando el modo de entender las lógicas de acción que involucran tanto al Estado, como a la Santa Sede. La propuesta consiste, por un lado, en dejar de pensar al Estado nacional como un actor cuyos intereses específicos se impusieron sobre los de otros actores de la política argentina (en este caso, las élites provinciales o los sectores del clero afines a Roma), para considerarlo -siguiendo los estudios reseñados más arriba- como un espacio donde esos actores tomaron decisiones y movilizaron recursos para dar forma a instituciones de escala nacional. Proponemos incluir entre esas instituciones a la Iglesia argentina.

Un cambio similar de perspectiva puede ensayarse sobre la influencia romana, y este es el segundo desplazamiento que se propone aquí. Así como la construcción del Estado nacional dejó de verse como el avance desde un centro hacia la periferia, estudios recientes sobre la consolidación de la autoridad papal destacan que ese fortalecimiento no fue promovido exclusivamente por la curia romana, sino que formó parte de los objetivos de movimientos y actores del catolicismo mundial en el marco de batallas culturales libradas a lo largo del siglo XIX a raíz de los procesos de formación de los Estados-nación. De esta manera, la romanización deja de pensarse como un avasallamiento de las expresiones locales del catolicismo por parte de la Santa Sede, para entenderse en cambio como un proceso multilateral que asumió formas diversas y particulares a cada espacio (Clark, 2003).

La estructura del artículo obedece al doble desplazamiento propuesto. En el primer apartado se vuelve sobre el tema del financiamiento del culto católico por parte del Estado nacional. La intención es indagar un fenómeno que ha sido poco observado y menos explicado todavía: las diferencias que existieron entre los montos aprobados por el Congreso y las partidas efectivamente giradas por el Poder Ejecutivo a las iglesias de la Confederación. ${ }^{5}$ Sugerimos que esas diferencias no solo dan cuenta de la distancia entre el optimismo voluntarista del gobierno y su cruda realidad económica, sino que además permiten estudiar la existencia de diversos actores dentro del Estado (los legisladores, los funcionarios que ocupaban las oficinas del Poder Ejecutivo, pero también, de forma menos directa, el clero de las provincias) que

\footnotetext{
5 El trabajo de Lida (2007) con el que se dialoga aquí no tiene en cuenta esta diferencia y considera los montos aprobados como si hubieran sido efectivamente erogados. Garavaglia (2010, pp. 233-234), en cambio, sí señala esta diferencia, pero lo hace en el marco de un estudio general de las rentas de la Confederación. Además, adjudica estas diferencias exclusivamente a la penuria económica fiscal, crónica en este período (agradezco a el/la evaluador/a que me sugirió la lectura de este artículo).
} 
gravitaron en el destino y el volumen de esos fondos. En el segundo se analiza la correspondencia que circulaba entre las oficinas pontificias y sus contactos de confianza en Sudamérica. Intentamos demostrar aquí que la intervención romana en la vida eclesiástica argentina no se limitó a la del enviado papal, y que en las decisiones tomadas por la Santa Sede para la provisión de las diócesis vacantes y la creación del obispado del Litoral tuvieron un peso importante los informes y criterios aportados por esos contactos locales.

\section{El sostén económico del culto entre el Congreso y el Ministerio}

El compromiso de sostener económicamente el culto Católico Apostólico Romano, sancionado en el artículo $2^{\circ}$ de la Constitución en 1853, iba en sintonía con el programa de construcción institucional del gobierno nacional. En el plano legal, esa decisión estaba estrechamente vinculada a la voluntad de conservar el ejercicio del patronato sobre la Iglesia católica, es decir, la facultad de presentar a los candidatos a obispos y demás beneficios eclesiásticos en el territorio argentino para su institución canónica por parte del Papa. En el núcleo de esta relación sobrevivía una lógica jurídica heredada de la colonia -y cuestionada para esos años por la Santa Sede y sus partidariosque hacía de la asistencia material a las iglesias por parte del poder civil un fundamento legal válido para incidir en su gobierno. ${ }^{6}$ Ello explica que ambas figuras, el sostén y el patronato, hayan formado parte del texto constitucional. También explica que el sostén no abarcara toda la estructura eclesiástica (por eso preferimos hablar de asistencia), sino aquellos espacios que el Estado nacional consideraba necesario controlar, como las autoridades diocesanas; u otros que también habían otorgado por tradición el derecho de patronato, como la evangelización -que ahora, además, se hacía más acuciante en la búsqueda de nuevas tierras allende la "frontera indígena"-. Los beneficios parroquiales, por ejemplo, no estaban incluidos. ${ }^{7}$ Sumado a ello, el interés por asistir económicamente a las estructuras eclesiásticas obedecía a un plan más amplio de construcción institucional. De allí que también se giraran fondos para la refacción de iglesias en las provincias: los templos no solo eran expresión material de la prosperidad de la comunidad y escenario de su vida cívica, sino también el ámbito donde se desarrollaban funciones de Estado tan

6 El tema fue muy desarrollado en Di Stefano y Zanatta (2000); Di Stefano (2011).

7 Desarrollo más extensamente este tema en Martínez (2013). No es casual que la asistencia nacional coincidiera en líneas generales con los espacios que financiaba el diezmo durante la colonia, que también era administrado por el rey en tanto patrono. 
importantes como el registro de los nacimientos y matrimonios. ${ }^{8}$

Estas medidas y el sensible crecimiento de los montos destinados al culto en cada presupuesto anual aprobado por el Congreso le permitieron concluir a Lida (2007) que el gobierno nacional había asumido la tarea de crear una Iglesia centralizada y piramidal, subordinada al poder político nacional, muy similar al modelo institucional galicano, profundizado tras la Revolución Francesa. ${ }^{9}$ Coincidimos con la autora en que la decisión de asistir económicamente a la Iglesia católica en la Confederación estuvo influida por una concepción galicana de la relación entre poder político y esfera eclesiástica, pero deseamos advertir que esa concepción parece haber sido más que un programa de gobierno. Fue, según entendemos, el supuesto con el que diferentes actores dentro del Estado negociaron la distribución de recursos económicos y, por lo tanto, el marco de diferentes políticas posibles.

Trataremos de demostrar esa afirmación estudiando con qué criterios se fueron modificando los montos destinados al culto en el presupuesto anual, desde la discusión del proyecto del Poder Ejecutivo en las cámaras legislativas, hasta las libranzas efectivas ordenadas por el Ministerio de Justicia, Culto e Instrucción Pública (MJCIP) y los reclamos que se originaron cuando estas últimas no coincidían con las cifras dispuestas por el presupuesto. Por desgracia, ese itinerario se ha conservado en los archivos de manera sumamente fragmentaria. El eslabón más frágil de la cadena son los registros del Ministerio, puesto que sus documentos se encuentran perdidos o dispersos entre el Archivo General de la Nación (AGN) en Buenos Aires y el Archivo del Ministerio de Relaciones Exteriores y Culto. A esa dificultad se debe sumar que, en el AGN, los legajos de esta repartición no siempre se encuentran correctamente catalogados. ${ }^{10}$ De allí que solo pudiéramos recuperar para los años 1857 y 1858 los reclamos mencionados y la justificación del MJCIP frente al incumplimiento de los pagos

\footnotetext{
8 Sobre la política religiosa de la Confederación, a las obras citadas de Lida debe agregarse el trabajo insoslayable de Auza (1979). Para un panorama más amplio de la relación entre construcción del Estado y la Iglesia argentinos remitimos nuevamente a Di Stefano y Zanatta (2000); Di Stefano (2011).

9 La autora contrasta este modelo con la política de la provincia de Buenos Aires de esos años, que habría tendido a dejar la construcción de la Iglesia en manos de la incipiente sociedad civil, de acuerdo con el modelo anglosajón. Di Stefano (2013) discute esta hipótesis sobre la base de registros diferentes a los del presupuesto. Por un lado, analiza debates parlamentarios donde se advierte la vitalidad que conservan las concepciones galicanas en la élite gobernante, por el otro, da cuenta de la importante proporción que alcanzan los fondos extraordinarios destinados por el Estado provincial a la Iglesia bonaerense.

10 Sobre la desaparición de los archivos del gobierno de la Confederación ver Garavaglia (2010, pp. 224, 225). Cabe señalar que, al menos en Culto, el panorama es un poco menos desalentador que el que presenta el autor, que no menciona la documentación del MJCIP utilizada aquí. Por otro lado, así como hemos dado con un legajo entero que contiene órdenes de pago del MJCIP para el período marzo 1860 a diciembre de 1861 (Sala X, 44-8-5. AGN, Buenos Aires) que no figura en el catálogo, pueden existir otros en la misma condición.
} 
que había dispuesto el Congreso Nacional desde 1856. Aunque ello impida describir con detalle el derrotero del presupuesto destinado al culto para todo el período de la Confederación, nos permite al menos indagar la lógica que operó en los diferentes espacios en que se fue definiendo dicha inversión.

Para analizar el presupuesto en estos años no solo es útil evaluar sus cifras, sino también los rubros en los que se distribuían los fondos y la proporción que le correspondía a cada uno. En este plano, entre 1855 y 1857 ocurrió un cambio muy significativo: mientras el presupuesto aprobado aquel año para 1856 asignaba una partida para cada provincia, en el de 1858 los rubros definidos obedecían a la estructura eclesiástica a nivel nacional: Obispados, Subvenciones Eclesiásticas y Gastos Eventuales del Culto. En cuanto a las proporciones, de uno a otro se tendió a concentrar los recursos en los gastos fijos de la jerarquía diocesana y a otorgar mayor nivel de discrecionalidad al Poder Ejecutivo para distribuir el resto.

El cambio no fue súbito y tuvo ciertas resistencias. La distribución por provincias de 1856 obedecía al criterio que había llevado al Gobierno Nacional Delegado a fijar por decreto en enero de 1854 una asignación para el culto que guardara proporción con lo recaudado en concepto de diezmo (recientemente suprimido) por cada provincia en el último quinquenio. ${ }^{11}$ Por eso, en muchos casos, las cifras del presupuesto eran las mismas -o muy similares- a las de las recaudaciones de diezmos que habían declarado las provincias un año atrás. Había, no obstante, excepciones significativas: aquellas provincias que eran cabecera de diócesis (Salta, San Juan y Córdoba) tenían una asignación superior al resto en el presupuesto de 1856, independientemente de los montos declarados. Aunque no contemos con el detalle desagregado de estas partidas, es altamente probable que ese aumento responda a la ley que había sido sancionada recientemente con las escalas de pensiones que debía pagar el Estado nacional a los beneficios de las catedrales. ${ }^{12}$ Ahora bien, en el año 1857, el criterio para definir los ítems del presupuesto cambió: en este caso, cada partida correspondió a una diócesis y no a una provincia. El monto total destinado a Culto se distribuyó entre los obispados del Litoral (cuya creación se promovía), Córdoba, Salta y Cuyo. ${ }^{13}$ Pero el cambio fue parcial. Mientras en

11 Decreto del 5 de enero de 1854. Registro Oficial de la República Argentina (RORA), Buenos Aires, 1882, tomo III, p. 94. De esa manera, el gobierno nacional se proponía compensar a las provincias por la supresión del diezmo que había dispuesto el Congreso Constituyente a fines de 1854, ver Auza (1981).

12 Ley 85 sancionada el 18 de agosto de 1856. Congreso Nacional. Acta de Sesiones de la Cámara de Senadores (ASCS), 1891, p. 364.

13 Litoral, $\$ 4.840$; Córdoba, $\$ 18.640$; Salta, $\$ 38.340$; Cuyo, $\$ 14.200$. Hemos tomado las cifras del informe del Ministerio de Hacienda publicado en El Nacional Argentino (ENA), 23 de junio de 1858, Paraná. Biblioteca Nacional, Sección Hemeroteca (BN-SE), Buenos Aires. 
algunos rubros las asignaciones tendieron a ajustarse a las dimensiones institucionales de las iglesias -como en el de las pensiones catedralicias-, en otros pareció regir el deseo de conservar cierta igualdad entre provincias. Así ocurrió cuando los diputados modificaron el proyecto original del Poder Ejecutivo, que otorgaba diferentes sumas a las provincias que no eran sede de obispado, para gastos de culto y reparación de templos. La cámara baja rechazó esas diferencias y sancionó en cambio un subsidio uniforme de \$2.000 para cada una, independientemente de las dimensiones de sus iglesias o las necesidades económicas que tuvieran. En esa sesión estaba presente el ministro del MJCIP, quien cuestionó la medida y propuso en cambio que se aprobara un monto general, cuya distribución sería definida por el Ministerio según lo que cada caso requiriera. ${ }^{14}$ Aunque en la cámara alta el proyecto original fue ratificado por Diputados, contó con los dos tercios necesarios para imponer los puntos reformados y el presupuesto del año 1857 mantuvo esa cuota igualitaria de fondos para las iglesias provinciales. ${ }^{15}$

Un año más tarde, cuando las cámaras discutieron el presupuesto para 1858, el progresivo abandono de la dimensión provincial en la distribución del presupuesto se profundizó más. La asignación de \$2.000 para cada provincia sin catedral que había sido defendida con tanto empeño por la cámara baja desapareció y, en cambio, se creó la partida denominada "Subvenciones Eclesiásticas", de \$16.650, que el MJCIP podría destinar a las iglesias provinciales en las proporciones que considerara convenientes. Esta novedad no pasó desapercibida para los diputados Pedro L. Funes y Genaro Feijóo, quienes reclamaron que, tal como había ocurrido el año anterior, se destinara un monto fijo a cada provincia. En esa ocasión, el ministro de JCIP, presente en la sala, encontró el apoyo necesario para imponer el criterio que el año anterior había fracasado. También se redujeron a una única partida los fondos destinados a las autoridades diocesanas, catedrales y seminarios. Bajo la genérica denominación "Obispados", se incluían ahora las cuatro diócesis que el año anterior estaban diferenciadas por partidas. Solo que, en este caso y a diferencia de las subvenciones eclesiásticas, las cámaras sí designaron sumas específicas para cada diócesis. La asignación ahora fue mucho más igualitaria: Córdoba, \$17.640; Salta, \$17.340; Cuyo, \$17.740; y el Litoral-que seguía siendo en 1857 una diócesis potencial, puesto que la Santa Sede aún no había dispuesto su creación y, por lo tanto, carecía de obispo, curia y cabildo eclesiástico- tenía

14 Sesión del 7 de septiembre de 1856. Congreso Nacional. Acta de Sesiones de la Cámara de Diputados (ASCD), 1891, pp. 466-468.

15 Sesiones del 19 y 27 de septiembre de 1856. ASCS, 1891, pp. 271-273 y 318-329. 
asignados \$5.056. La tercera partida también supuso una mayor libertad para el Ministerio, fueron $\$ 4.000$ correspondientes a "Gastos Eventuales" que su nombre podría destinar a gastos no previstos. Aquí los diputados pusieron un límite al Poder Ejecutivo, y redujeron la cifra original a la mitad. ${ }^{16}$

Como anticipamos, los montos del presupuesto no indican lo efectivamente invertido en culto por el fisco nacional. En primer lugar, porque a la par de esa ley las cámaras solían votar otras complementarias que disponían auxilios puntuales para la estructura eclesiástica. En segundo término porque, como lo demuestran los informes de gastos del Ministerio de Hacienda (las denominadas Cuentas de Inversión), los montos realmente erogados, al menos para 1857 y 1858, fueron inferiores a los presupuestados. La diferencia no es menor. Según las Cuentas de Inversión para 1857, el ahorro fue del 54,6\%, considerando la ley del presupuesto y complementarias, mientras que en 1858 ascendió al $62,46 \%$. Esas reducciones fueron presentadas como un logro importante por el Ministro de Hacienda, fundamentalmente porque compensaban el alto nivel de deuda que había generado el déficit del año $1856 .{ }^{17}$ Lo que nos interesa aquí es conocer con qué criterios se decidió ese ahorro.

La correspondencia intercambiada entre el MJCIP y las autoridades eclesiásticas de la Confederación, que se dirigían a Paraná reclamando el pago de las partidas del presupuesto, nos da una pista para conocer esos criterios. Los reclamos eran de dos tipos: se adeudaban pagos desde el año 1856 y, cuando se liquidaban, las cifras eran menores a lo establecido por la ley. ${ }^{18}$ Estas quejas se hicieron tan frecuentes, que el ministro formalizó una respuesta que repetía casi sin variaciones en todos los casos:

16 Sesiones del 25 y 29 de agosto de 1857. ASCD, 1891, pp. 231 y 246-248. El presupuesto finalmente aprobado puede consultarse en ASCS, 1857, p. 468.

17 La deuda del año 1856 en ENA, 14 y 15 de junio de 1858. Las cuentas de inversión de 1857 en $E N A, 23$ de junio de 1858; las de 1858 en ENA, 13 y 14 de junio de 1859. BN-SE, Buenos Aires.

18Así se desprende de la correspondencia intercambiada entre las autoridades eclesiásticas de la Confederación y el MJCIP, conservadas en el legajo 44-8-3 de la Sala X del AGN, que reúne esa correspondencia desde febrero de 1857 a diciembre de 1858. Las protestas son varias, citamos a continuación algunos casos que dan cuenta de la extensión geográfica del problema. El 3 de abril de 1857 el MJCIP se dirigía al vicario foráneo de Jujuy, Escolástico Zegada, explicándole que solo autorizaría gastos que el vicario justificara dando cuenta de la estructura a solventar (Sala X, 448-3, pp. 32-33); hay otra carta, también del MJCIP a Zegada fechada el 16 de abril de 1857, Sala X, 44-8-3, p. 37. En 1858 se debía a la Iglesia de Santa Fe partidas correspondientes a 1857, ver nota del MJCIP al Delgado Eclesiástico de Santa Fe, Paraná, 25 de febrero de 1858, Sala X, 44-8-3, p. 126. Con el mismo tenor: nota del MJCIP al obispo de Salta, Paraná, 13 de enero de 1858, Sala X, 44-8-3. p. 108, y MJCIP al Delegado Eclesiástico de Corrientes, Paraná, 24 de febrero de 1858, Sala X 44-8-3, p. 120 y Sala X, 44-9-6, p. 15. Sobre montos atrasados desde 1857 también respondía el MJCIP al gobernador del obispado de Córdoba, Paraná, 20 de abril de 1858 y el 14 de julio de 1858. Sala X, 44-8-3, pp. 145 y 176 respectivamente. AGN, Buenos Aires. 
“El espíritu de esta Ley [el presupuesto] no tiende á poner al Gob.no en la obligacion de pagar integras las asignaciones que en ella se votan; solo importa una autorizacion que le dá el Congreso para poder invertir hasta el monto de esas sumas en los diversos gastos que ocurren en los ramos de la Adm.cion.... Por la misma razon, pues, no concede á las Iglesias el derecho de exijir toda la cantidad que se haya votado para sus gastos, debiendo solo librarse las que sean necesarias para atender los gastos estrictamente indispensables." ${ }^{\prime 19}$

Así, los montos que recibían las iglesias de la Confederación dependían, en última instancia, de las planillas de beneficiados declarados por sus autoridades, o de gastos de mantenimiento concretos, igualmente justificados. ${ }^{20}$

En definitiva, mientras algunos diputados y senadores buscaron incrementar la participación de las iglesias provinciales en la inversión estatal y limitar el margen de decisión del Poder Ejecutivo, el MJCIP dispuso de los instrumentos administrativos de su cartera para obrar en sentido inverso. En tanto ambos criterios tuvieron expresión en las disposiciones del Estado nacional, las autoridades eclesiásticas locales encontraron cierto espacio para gestionar recursos del erario. Lo que todos tenían en común, incluidos los sacerdotes que reclamaban el cumplimiento puntual y completo de las partidas votadas en el Congreso, era la convicción de que el Estado debía, como lo establecía la Constitución, sostener materialmente el culto. En el apartado siguiente veremos que también la Santa Sede y sus partidarios locales compartían este principio, y se valieron de él para incidir en las políticas nacionales de financiamiento del culto. Asimismo, definieron los tiempos y, en alguna medida, los protagonistas de la construcción institucional de la Iglesia argentina.

\section{Agentes y modos de la romanización}

En el artículo con el que dialogaremos en esta sección, los intereses romanos aparecen identificados con la acción del delegado apostólico Marino Marini (Lida, 2006). Su llegada a Paraná a raíz de las negociaciones de Salvador Jiménez, enviado en misión oficial a Roma por el gobierno de Urquiza, constituía una victoria sobre el gobierno porteño, con el que el de la Confederación

19 Carta del titular del MJCIP al Delegado Eclesiástico de Santa Fe, Paraná, 27 de junio de 1857, Sala X, 44-8-3, p. 64.AGN, Buenos Aires.

20 El procedimiento de confección de planillas fue explicado a las autoridades de las diferentes jurisdicciones eclesiásticas. Ver nota del MJCIP al gobernador del obispado de Córdoba, José G. Baigorrí, Paraná, 20 de abril de 1858, Sala X, 44-8-3, p. 145. AGN, Buenos Aires. 
venía manteniendo una encarnizada batalla diplomática. ${ }^{21}$ Pero además, la presencia de Marini fue funcional a las intenciones del gobierno urquicista de contrarrestar la influencia que el obispo de Buenos Aires, Mariano Escalada, tenía sobre las iglesias de las provincias del Litoral que pertenecían a su diócesis. Miranda Lida advierte muy lúcidamente cómo Marini comenzó a cumplir el rol no solo de emisario papal, sino de arzobispo de hecho para las iglesias de la Confederación. Según la autora, una vez que la secesión bonaerense finalizó y las autoridades políticas de la nación se alojaron en Buenos Aires, la presencia de un emisario papal como máxima autoridad de la Iglesia argentina obstaculizaba el proyecto de centralizar esa Iglesia, subordinada al poder estatal. Mucho más conveniente resultaría convertir en arzobispo al obispo de Buenos Aires. De esa manera, la máxima autoridad eclesiástica de la nación quedaría bajo la órbita del poder civil -que ejercía el patronato-, y el papel de Roma sería relegado al de "simple actor de reparto" (Lida, 2006, p. 28).

Si bien esta interpretación tiene la virtud de echar luz sobre el rol que cumplió Marini durante su estancia en Paraná, presenta el problema de confundir la suerte que corrió la autoridad romana en la Argentina con la del delegado apostólico. Esa confusión es heredera, como se anticipó en la introducción, de una concepción del proceso de romanización entendido como un cambio impulsado de manera unilateral por el papado. Este artículo propone incluir a actores locales -que aquí denominaremos ultramontanos- como partícipes de la romanización en Argentina. Se trata de un conjunto heterogéneo (y de dudosa cohesión interna) que compartía el objetivo general de colocar a la religión católica como matriz moral y cívica de la nación que se estaba construyendo. Para garantizarlo, sus miembros consideraban necesario fortalecer la imagen de la Iglesia como sociedad perfecta, esto es, esencialmente autosuficiente y gobernada por sus propias autoridades, encabezadas por el Papa. Este embrionario grupo ultramontano había surgido lentamente durante las décadas anteriores, primero estimulado por el debate religioso en torno a la sanción de leyes de reforma eclesiástica y libertad religiosa, y luego por el contacto cada vez más fluido con emisarios papales, cuya presencia en América se hizo permanente a partir de 1830, cuando la Santa Sede instaló en Río de Janeiro una legación diplomática (Di Stefano, 2004, pp. 155-192; Martínez, 2014a). Aunque este sector-compuesto por eclesiásticos, pero también por seglares- actuaba muy vinculado a las oficinas romanas y sus delegados, no dependía de la presencia

21 Marino Marini llegó a Paraná en febrero de 1858, luego de haber actuado como encargado de negocios de la Santa Sede en Río de Janeiro desde 1853 a 1856. Fue el primer diplomático romano reconocido oficialmente por un gobierno argentino. Antes que él habían pisado suelo rioplatense las misiones apostólicas encabezadas por Giovanni Muzi en 1824 y Ludovico Besi en 1851, pero ninguno de ellos recibió el reconocimiento oficial de los gobiernos del momento. 
de un delegado apostólico en territorio argentino para operar. ${ }^{22}$

En los apartados siguientes se fundamenta esta afirmación. En el primero se destaca la importancia que tenía el obispo (y futuro arzobispo) de Buenos Aires, Mariano Escalada, como referente ultramontano y eslabón clave entre las autoridades romanas y las iglesias argentinas. En el segundo se analizan los informes que algunos miembros de este grupo remitieron a Roma sobre los candidatos a ocupar las diócesis argentinas vacantes. En tercero se estudian las medidas tomadas por la Santa Sede para evitar o aplazar el nombramiento de aquellos candidatos presentados por el gobierno que no habían merecido la aceptación de los informantes locales. Ese protagonismo del sector ultramontano local permite pensar que el alejamiento del delegado apostólico en la década de 1860 y la elevación precisamente de Escalada como arzobispo de Buenos Aires y metropolitano de las demás diócesis de la nación no fueron en desmedro de la presencia romana en la incipiente Iglesia argentina.

\section{2-a. Escalada}

Para 1853, Mariano Escalada tenía ya una larga trayectoria en la vida eclesiástica rioplatense. Había oficiado como secretario del obispo de la diócesis de Buenos Aires, Mariano Medrano, y luego como su obispo auxiliar a partir de 1835. La confianza que habían depositado en él los representantes de Roma en América, desde la instalación de la nunciatura en Río de Janeiro en 1830, lo convirtió en el principal consejero de la Santa Sede durante los primeros años de contacto directo del poder papal con las iglesias del Río de la Plata (Ayrolo, 1996; Martínez, 2014a). Su defensa de los jesuitas durante el conflicto que mantuvieron con Juan Manuel de Rosas marcó el inicio de un distanciamiento con el gobierno de Buenos Aires que se fue profundizando hasta ubicarlo como un abierto opositor durante la década de 1840. Con esas credenciales, y fallecido en 1851 el obispo Medrano, Escalada era uno de los principales candidatos

\footnotetext{
22 Carecemos de estudios que nos informen satisfactoriamente sobre los miembros de este espacio ultramontano en las décadas centrales del siglo XIX, más allá de trabajos de corte celebratorio o militante dedicados a algunas figuras relevantes (Tonda 1952, 1961). Más valiosas para nuestro trabajo son las reflexiones de Roberto Di Stefano (2004, pp. 155-192) sobre la corriente de pensamiento que inspira a este grupo y que el autor prefiere llamar "intransigente". Se han comenzado a estudiar las trayectorias de algunos de sus miembros desde perspectivas más actualizadas (Ayrolo, 2002; Ayrolo y Mazzoni, 2013). En todos los casos, las investigaciones privilegiaron el estudio del clero. Sabemos poco también de la participación de seglares en este espacio ultramontano, excepción hecha de Félix Frías, aunque siempre desde un enfoque clásico (por ejemplo, Tonda, 1956). He comenzado a estudiar los vínculos que permitieron la circulación de ideas e información entre estos personajes, de allí la idea de una cierta cohesión, aunque débil (Martínez, 2014a). Un panorama más general en Martínez (2014b).
} 
de Roma y del sector ultramontano a ocupar la sede vacante. ${ }^{23}$ Su oposición al rosismo también lo hacía aceptable a la élite y al gobierno porteño.

De allí que, más allá de la llegada de un representante papal a la Argentina, la presencia romana parecía estar garantizada en el país, según los partidarios de la Santa Sede, por la permanencia de Mariano Escalada en el obispado de Buenos Aires. Es más, el paso lógico, afirmaban los ultramontanos, era elevar su silla a metropolitana para asegurarle una superioridad jerárquica sobre las demás diócesis argentinas. ${ }^{24}$ Esa fue también la opinión del mismo Marini en 1856, cuando oficiaba como encargado de negocios de la Santa Sede en Río de Janeiro. En despacho a la Secretaría de Estado vaticana observó que la solución de los problemas que surgían de los interregnos en las diócesis argentinas era elevar al obispo de Buenos Aires a metropolitano una vez que esa provincia se hubiera incorporado al ámbito nacional. Así se podría conservar mejor la disciplina eclesiástica y se recuperaría el orden de las apelaciones. ${ }^{25}$ Pocos meses después, al desarrollar su proyecto, Marini afirmaba que el mejor candidato para ocupar la sede metropolitana era Escalada, que había:

\begin{abstract}
"desplegado una decisión y una energía no ordinaria regulada sin embargo de la necesaria prudencia.... Con su virtud y afabilidad se ha ganado el amor y la estima universal, y es respetado y aceptado por el Gobierno a pesar de la firme resistencia que ha opuesto a sus extrañas pretensiones. Con estos medios, y ayudado por el tiempo, podrá conducir a buen término las útiles reformas que se ha propuesto porque está aún en la edad fresca de cincuenta y siete años, y es sano y vigoroso." 26
\end{abstract}

23 El sacerdote irlandés Antonio Fahy, residente en Buenos Aires, insistía con la necesidad de nombrarlo obispo de esa diócesis. Carta de Fahy al arzobispo de Armash. Buenos Aires, 20 de enero de 1850. Argentina I, fascículo 31, pos. 68, folios 91-95. Archivio della Congregazione per gli Affari Ecclesiastici Straordinaric (AES), Ciudad del Vaticano.

24 Así lo expresó a Marini el exministro de Relaciones Exteriores de la Confederación, Facundo Zuviría, antes de la designación de aquél como delegado apostólico en Argentina. Para Zuviría, Escalada podría suplir al nuncio apostólico que se venía solicitando desde 1853, puesto que ese sacerdote "bien merece ser el ojo, el organo, el representante de S. Santidad en estas Republicas desorganizadas en todo con 45 años de revolucion y anarquia." Carta de Zuviría a Marini. Montevideo, $1^{\circ}$ de mayo de 1856, fascículo 283, folio 128. Archivio Segreto Vaticano, Achivio Nunziatura Brazile (ASV, ANB), Ciudad del Vaticano.

25 Despacho de Secretario de Estado de la Santa Sede. Río de Janeiro, 12 de mayo de 1856. Argentina I, fascículo 48, pos. 98, folios 70-75. AES, Ciudad del Vaticano. Sobre el problema de las apelaciones, que se arrastraba desde 1810, ver Di Stefano (2004).

26 Despacho de Secretario de Estado de la Santa Sede. Río de Janeiro, 13 de septiembre de1856. Argentina I, fascículo 48, pos. 98, folios 100-102v; la cita en folio 100. AES, Ciudad del Vaticano. 
El tiempo y sus virtudes eran indispensables, pero no suficientes para encarar las reformas que necesitaba la Iglesia argentina. Era necesario además que Escalada fuera acompañado en las otras diócesis por obispos que compartieran esos objetivos. Veremos a continuación los mecanismos mediante los cuales el sector ultramontano buscó incidir en el perfil de las autoridades eclesiásticas que deberían secundar a Escalada en la implementación de esas reformas.

\section{2-b. Las listas para los obispados}

Tanto para el gobierno como para la Santa Sede y sus partidarios urgía instituir obispos para las tres diócesis de la Confederación, que estaban todas vacantes. ${ }^{27}$ En 1855, poco después de conformado el Congreso Nacional y siguiendo el procedimiento establecido por la Constitución, el Senado confeccionó una terna de candidatos para cada silla episcopal. A partir de esas ternas, el Poder Ejecutivo seleccionó a los primeros de cada una y los presentó al Papa para su institución canónica. ${ }^{28}$ Además, desde la llegada en 1854 de la misión oficial de Salvador Jiménez a Roma, el gobierno nacional negociaba la creación de una diócesis con sede en Paraná, que independizaría en el plano eclesiástico a las provincias de Entre Ríos, Santa Fe y Corrientes, pertenecientes en ese momento al obispado de Buenos Aires. ${ }^{29}$

Desde el punto de vista romano, la situación no era sencilla. La Santa Sede rechazaba el argumento del gobierno para ejercer el patronato, según el cual el Estado argentino había heredado esa facultad de la monarquía española, junto con la soberanía sobre el territorio. Ello no significaba que el Papa se negara a conceder el uso de esa facultad a los gobiernos hispanoamericanos, pero para hacerlo, argumentaba, era necesario firmar un concordato donde el poder civil otorgara ciertas garantías y privilegios a la religión e Iglesia católica. ${ }^{30}$ En vistas a ese acuerdo, los cardenales de la Congregación de Asuntos Eclesiásticos Extraordinarios (AES, por sus siglas en italiano), que era la

\footnotetext{
27 Ver el discurso de Urquiza en la apertura de sesiones del Congreso Nacional en octubre de 1854. ENA, 9 de noviembre de 1854. BN-SE, Buenos Aires. En el mismo sentido, despacho de Secretario de Estado de la Santa Sede. Río de Janeiro, 12 de mayo de 1856. Argentina I, fascículo 48, pos. 98, folios 70-75. AES, Ciudad del Vaticano.
}

28 Decreto del 21 de agosto de 1855. RORA, tomo III, p. 242.

29 Instrucciones extendidas al agente confidencial, Salvador Jiménez. Paraná, 8 de enero de 1854, publicada por Centeno (1909).

30 Así ocurrió durante esa década con los concordatos firmados por los gobiernos de Centroamérica, mediante los cuales el Papa concedía el patronato al poder político mientras éste se comprometiera a: respetar la libre comunicación entre Roma y el clero local, asegurar la educación religiosa obligatoria y controlada por los obispos, sostener económicamente el culto y los seminarios, y permitir la censura eclesiástica a la prensa (Picado, 1993). La legislatura boliviana, por su parte, trató un concordato muy similar en 1851 (Ministerio de Relaciones Exteriores de Bolivia, 1883). 
encargada de aconsejar al Papa en materia de política exterior, no deseaban contrariar abiertamente las pretensiones del gobierno de la Confederación.

Es comprensible, en este contexto, que el proceso fuera lento. Recién a partir de 1858 las diócesis fueron provistas, y en 1859 se creó el obispado del Litoral. De los candidatos originales presentados por el gobierno, solo dos se consagraron finalmente obispos. Los restantes, José Leonardo Acevedo, electo obispo del Litoral, y José Gregorio Baigorrí, de Córdoba, murieron esperando sus bulas de institución. Durante esos años, Roma se tomó su tiempo para recabar la información necesaria sobre los sacerdotes argentinos dignos de la mitra, y se tomó más aún para dejar morir a los que parecían no serlo. Esa conclusión se desprende de la documentación conservada en el Archivo de la Secretaría de Estado de la Ciudad del Vaticano. Allí se reúnen la correspondencia enviada a Roma por Marino Marini y las actas de la Congregación de AES. Nos detendremos en el estudio de esa documentación durante el período en que Marini residió en Río de Janeiro, previamente a su llegada a la Argentina como delegado apostólico. Ello nos permitirá analizar, por un lado, quiénes eran los principales consejeros de Roma para el nombramiento de obispos, y por el otro, qué características se consideraban adecuadas para un obispo y cuáles no.

Un año antes de la presentación oficial de los candidatos a obispo por parte del gobierno, llegó a Roma un informe anónimo donde se ofrecía una lista de los sacerdotes confiables y otra de los inadecuados para gobernar las diócesis argentinas. A raíz de ello, la Secretaría de Estado pidió a Marini que contrastara esa información con sus contactos de confianza en la Argentina. El diplomático se dirigió a Escalada solicitando un informe, quien lo remitió entre noviembre y diciembre de $1854 .^{31}$ En diciembre de 1855, pocos meses después de que Urquiza emitiera el decreto de presentación, y a instancias de la Congregación de AES, Marini comenzó a solicitar y a recopilar informes no solamente sobre los candidatos presentados por el gobierno, sino también sobre otros sacerdotes que, aun estando excluidos de las listas, podrían ser nombrados motu proprio por el Papa como obispos auxiliares. La intención era colocar a personas de confianza en aquellas diócesis donde el candidato oficial no lo fuera. Se buscaba, además, asegurar la sucesión dentro del grupo de sacerdotes ultramontanos, porque se especulaba que, cuando la diócesis quedara vacante, el obispo auxiliar tendría más chances de formar parte de la próxima terna. ${ }^{32}$

En este caso, el principal interlocutor de Marini fue Facundo Zuviría,

31 Argentina I, fascículo 46, pos. 84. AES, Ciudad del Vaticano.

32 Despacho de Secretario de Estado de la Santa Sede. Río de Janeiro, 12 de mayo de 1856. Argentina I, fascículo 48, pos. 98, folio 52-52 v. AES, Ciudad del Vaticano. 
quien había tenido un papel importante en la primera etapa de la política eclesiástica urquicista como ministro de Relaciones Exteriores, pero que ya en 1856 se encontraba distanciado del gobierno y asilado en Montevideo, "hostilizado, y perseguido [según denunciaba] por una Logia Masonica establecida en el Paraná en torno del Gobierno, o del General Urquiza a quien ha dominado de un modo que carece de accion y voluntad propia". ${ }^{33}$ Otro contacto fundamental fue, nuevamente, Escalada, cuyo consentimiento, por lo demás, parece haber sido determinante para la erección de la nueva diócesis del Litoral. ${ }^{34}$ Sobre los informes de Zuviría y Escalada, Marini confeccionó finalmente tres listas. Una fue la de las ternas presentadas por el gobierno; la segunda nómina estaba integrada por los sacerdotes que, sin estar en las ternas, eran a su criterio buenos candidatos a la mitra; la tercera, finalmente, estaba formada por los eclesiásticos que podrían ser nombrados por la Santa Sede como obispos auxiliares allí donde fuera necesario. ${ }^{35}$ En todos los casos, los nombres fueron acompañados de una breve relación de méritos (o deméritos) de cada candidato.

¿Cuáles eran las virtudes que se requerían, según estos informantes, para ser nombrado obispo? ¿Y cuáles los defectos que colocaban a un eclesiástico entre los no recomendables? Para el informante anónimo que mencionamos en primer lugar, los méritos fundamentales de los recomendados eran: poseer hábitos moralmente irreprochables, haber rechazado la libertad de cultos declarada en la Constitución nacional y gozar de alta estima en su comunidad. ${ }^{36}$ Escalada mencionó también estos puntos en sus informes, pero se preocupó particularmente por detallar el nivel de formación de los candidatos. Solo en un caso -el del secularizado español Miguel Vidal, párroco de Paraná-, Escalada

33 Despacho de Marini a la Secretaría de Estado. Río de Janeiro, 14 de diciembre de 1855. Argentina I, fascículo 48, pos. 98. AES, Ciudad del Vaticano. Carta de Facundo Zuviría a Marini. Montevideo, $1^{\circ}$ de mayo de 1856, fascículo 283, folios 124-128. ASV-ANB, Ciudad del Vaticano. El informe de Zuviría fue publicado por Bruno (1980). Agradezco a uno/a de los/as evaluadores/as anónimos/as esta información. Sobre el desplazamiento de Zuviría trata con más detalle Lanteri (2013b, pp. 159-161).

34 Carta de Escalada a Marini. Buenos Aires, 10 de abril de 1856. Argentina I, fascículo 48, pos. 98, folios 92-93. AES, Ciudad del Vaticano.

35 Por cuestiones de espacio obviamos las listas de los ternados, que pueden consultarse en ASCS del 27 de julio de 1855 y en RORA, tomo III, p. 242. Fuera de las ternas, los recomendados eran: José Saturnino Allende (a quien recomienda además como auxiliar de Córdoba), Eusebio Agüero, José Vicente Agüero, Genaro Carranza, José V. Ramírez de Arellano, Isidoro Fernández, Genaro Feijóo, Escolástico Zegada, Miguel Aldurralde, Wenceslao Achával, Mamerto Esquiú, José Gelabert, Ildefonso Fernández Medrano, José Rolón, Miguel Vidal. Como auxiliares recomendaba, además de Allende, a Manuel Pérez para Salta y a Timoteo Maradona para Cuyo. Despacho de Secretario de Estado de la Santa Sede de la Santa Sede. Río de Janeiro, 12 de mayo de 1856. Argentina I, fascículo 48, pos. 98, folios 76-85v. AES, Ciudad del Vaticano. Las referencias mencionadas adelante corresponden a estos documentos.

36 Los recomendados eran: T. Maradona, J. S. Allende, M. Aldurralde, M. Pérez, J. Colombres, J. L. Acevedo, M. Vidal, José Manuel Figueroa. Los no recomendados: G. Feijó, G. Baigorrí, Juan José Álvarez, Manuel Antonio Castellanos, Juan Prieto, Gerónimo Becchi. 
elogió la "contracción a su ministerio". Zuviría también hizo hincapié en la moralidad, instrucción y desempeño pastoral de los candidatos. Además, consideraba como virtudes la buena salud o la juventud de los recomendados, dotes importantes en esa coyuntura de futuro incierto, donde era preciso garantizar un episcopado prolongado para aquellos sacerdotes que fueran confiables. En las listas de Marini, por último, también se destacaban estos méritos, y se señalaba además como virtud el hecho de ser aceptado por el gobierno. Solo en un caso (el de Timoteo Maradona, de San Juan) se destaca su resistencia a pretensiones jurisdiccionales del poder político.

Con respecto a los puntos negativos que podían perjudicar a los candidatos, el más recurrente era el de haber aceptado la libertad de cultos. Otra característica que restaba en la consideración era la edad avanzada o un estado de salud muy deteriorado. En los informes de Escalada, la escasa formación del primer ternado para la diócesis del Litoral, José Leonardo Acevedo, o la dudosa ortodoxia del candidato al obispado de Córdoba, José Gregorio Baigorrí, constituían también obstáculos para llegar a la mitra. ${ }^{37}$ Lamentablemente, el carácter por lo general escueto de los informes nos priva de detalles útiles para reconstruir mejor los criterios de estos informantes (por ejemplo, en ningún momento se aclara en qué consistía la heterodoxia de Baigorrí). Sin embargo, quizás ese mismo laconismo indique que el perfil deseado no era demasiado claro incluso para los interlocutores de este diálogo. De todas maneras, es curioso que en el aspecto doctrinal se privilegiara mucho más claramente el rechazo a la libertad de cultos que la defensa de las prerrogativas papales de gobierno eclesiástico. Quizás esto esté en consonancia con la postura coyuntural de Roma en los años de 1850 respecto del patronato, señalada más arriba.

Es muy significativo que, a pesar de considerar escasa la información que habían remitido sus contactos en la Argentina, la Santa Sede haya actuado siguiendo sus opiniones y criterios. ${ }^{38}$ Ello nos habla del carácter necesariamente mediado de la intervención papal o, mejor dicho, de la multiplicidad de actores que intervenían a la hora de definir la política romana en la Argentina. ${ }^{39}$ Veremos a continuación cuál fue esa política en el caso de la provisión de las diócesis de la Confederación.

37 Baigorrí "es muy viejo, y de ideas muy raras; por ambas razones no se le considera á proposito p.a Prelado". Carta de Escalada a Marini, sin fecha. Argentina I, fascículo 46, pos. 84, folios 15 a 16v. AES, Ciudad del Vaticano. Bruno (1980, p. 479) fecha esta carta, que consultó en el ASV, el 26 de diciembre de 1854.

38 La falta de informes más detallados le era sensible al Papa, que en audiencia de la Congregación de AES ordenó pedir más datos sobre los candidatos (Audiencia del 28 de noviembre de 1855. Argentina I, fascículo 48, pos. 98. AES, Ciudad del Vaticano).

39 Ayrolo (1996) advirtió ya el peso de esta mediación para décadas anteriores. 


\section{2-c. Los tiempos romanos}

En el clima de negociación que caracterizó a las relaciones entre la Santa Sede y la Confederación, la presentación por parte del gobierno de dos candidatos calificados negativamente por los informantes de Marini ponía a prueba la diplomacia romana. Se trataba, como vimos, de José Gregorio Baigorrí, pero particularmente de José Leonardo Acevedo, sobre quien se habían recibido varias advertencias acerca de su muy escasa formación. Ni siquiera Zuviría, quien se manifestaba abiertamente amigo del sacerdote, se atrevía a desmentir los informes que daban cuenta de este defecto..$^{40}$ En razón de la importancia del obispado que debía gobernar (uno nuevo que coincidiría en su sede con la capital provisional de la República) la primera decisión de la Congregación fue encargarle a Marini que persuadiera a Urquiza de presentar otro candidato. El diplomático contestó que era demasiado tarde: el gobierno argentino ya había hecho pública su presentación y no lo creía dispuesto a reconocer abiertamente que la Santa Sede tenía capacidad de veto en este asunto. Sería más conveniente, aconsejó Marini, aplazar la erección de la diócesis, exigiendo garantías como la dotación del obispo, del Cabildo Eclesiástico y del seminario, y la construcción de una catedral digna. Así se ganaría tiempo hasta que finalizara el mandato de Urquiza y con un nuevo gobierno podrían negociar más fácilmente la presentación de un candidato aceptado en Roma. En la sesión de julio de 1856, la Congregación decidió seguir el consejo de Marini y retrasar, no solo la creación de la diócesis del Litoral, sino la provisión de todas las otras hasta que el gobierno asegurara el sustento material de la estructura diocesana: estipendios del obispo, Cabildo Eclesiástico y curia, y fondos para el seminario conciliar. ${ }^{41}$

Las autoridades de la Confederación acusaron recibo de esta exigencia. En octubre de 1856, el gobierno dispuso la formación de dos comisiones integradas por vecinos notables, encargadas de recaudar fondos para la construcción de la catedral de Paraná. ${ }^{42}$ Además, la cantidad de miembros del proyectado Cabildo Eclesiástico de esa ciudad y el nutrido cuerpo de beneficiados catedralicios que se comprometió a sostener el gobierno, dan cuenta del eco

\footnotetext{
40 Consultado por Marini sobre este punto, Zuviría confirmó los temores romanos. Despacho del Secretario de Estado de la Santa Sede. Río de Janeiro, 12 de mayo de 1856. Argentina I, fascículo 48, pos. 98, folio 79. AES, Ciudad del Vaticano.

41 Despacho de Secretario de Estado de la Santa Sede. Río de Janeiro, 13 de mayo de 1856. Argentina I, fascículo 48, pos. 98, folios 94-95.Actas de la Congregación de AES, mismo expediente, folio 96, 96v. AES, Ciudad del Vaticano.

42 RORA, tomo IV, p. 426.
} 
que las pretensiones romanas tuvieron en los despachos de Paraná. ${ }^{43}$ Por último, fue sancionada una ley en septiembre de 1858 que disponía la creación de un seminario conciliar en cada diócesis dotado por el gobierno nacional. ${ }^{44}$ Debemos matizar entonces la afirmación de que el esfuerzo económico hecho por el Estado en estos rubros obedecía exclusivamente a la voluntad de construir una iglesia a su medida (Lida, 2006). En esa construcción la Santa Sede ponía sus condiciones y moldeaba también la forma de la Iglesia argentina.

Más subrepticia fue su influencia en los casos de los cuestionados Baigorrí y Acevedo. Los plazos prolongados ex profeso por la Congregación de AES resultaron demasiado largos para la salud de ambos sacerdotes, quienes murieron antes de poder ser instituidos canónicamente por el Papa. No fue necesario entonces esperar un cambio de gobierno. Para la diócesis de Córdoba, el Poder Ejecutivo presentó a José Vicente Ramírez de Arellano en reemplazo de Baigorrí. Ramírez de Arellano figuraba en la lista de los recomendados por Marini y no había formado parte de las ternas originales. Contando entonces con este antecedente, el Papa se tomó solo cuatro meses para instituirlo, mientras que el trámite de Baigorrí había demorado más de tres años (Bruno, 1974, pp. 133-136). Algo similar ocurrió con la diócesis del Litoral. Tras el fallecimiento de Acevedo, el presidente Urquiza presentó como candidato a obispo del Litoral a Luis Gabriel Segura y Cubas. Aunque en este caso la demora fue un poco mayor debido a que primero era necesario crear la diócesis, no pasó un año entre el decreto de presentación y la bula de institución, expedida el 21 de junio de 1859 (Segura, 1964, pp. 245-259). Segura también figuraba entre los recomendados. Había dado buenas referencias de él Zuviría, y Marini en su informe consignaba que era de una familia notable, y muy instruido, aunque no tuviera grados académicos. Se destacaba por haber fundado en Catamarca un colegio que hacía las veces de seminario diocesano. ${ }^{45}$

\section{Conclusión}

Hemos visto hasta aquí cómo, en las primeras etapas de la formación de la Iglesia argentina, el Estado nacional se desdibuja como actor monolítico y

43 La gravitación de la Santa Sede en estas medidas se ve confirmada en el despacho girado por el MJCIP a su par de Relaciones Exteriores el 7 de agosto de 1857, en el que le insiste que se le remita al Sumo Pontífice la ley sancionada por el Congreso con los sueldos que corresponden a obispos y demás dignidades de todas las diócesis, existentes y por crearse, para que el Papa acceda a erigir el nuevo obispado. Sala X, 44-8-3, p. 82. AGN, Buenos Aires.

44 RORA, tomo IV, pp. 149-150. Sobre los proyectos locales para erigir seminarios y las demoras para concretarlos trata Tonda (1957, pp. 33-43).

45 Despacho del Secretario de Estado de la Santa Sede. Río de Janeiro, 13 de mayo de 1856. Argentina I, fascículo 48, pos. 98, folio 79. AES, Ciudad del Vaticano. 
la Santa Sede difícilmente puede ser considerada el único agente romanizador. Las piezas parecen encajar mejor si se piensa que el incipiente aparato estatal y un todavía más embrionario círculo de eclesiásticos y laicos vinculados a Roma funcionaron como espacios donde confluyeron e interactuaron los actores concretos que participaron de ese proceso. Las formas de interacción en esos espacios combinaron la negociación con la utilización de ventajas relativas según el ámbito donde se tomaran las decisiones. Quienes buscaban fortalecer las iglesias locales consiguieron en el Congreso canalizar hacia ellas recursos del Estado nacional, al menos durante los primeros años. El Poder Ejecutivo, por su parte, modificó las cifras del presupuesto disminuyendo en la práctica los fondos destinados al culto según criterios administrativos aplicados desde las oficinas ministeriales. Las condiciones que estableció la Santa Sede para proveer diócesis y crear una nueva también influyeron, como vimos, en las partidas y leyes nacionales que asignaban recursos a la Iglesia. Los requisitos romanos, a su vez, surgían de informes y sugerencias de su representante en América, pero también de contactos locales como Escalada y Zuviría. Eran ellos quienes, además de evaluar a los candidatos, aconsejaban a Roma sobre los puntos que podían negociarse con el gobierno y en qué aspectos las autoridades de la Confederación no estarían dispuestas a ceder.

El desarrollo y el resultado final de esta complicada urdimbre de negociaciones e intereses no están estudiados aún satisfactoriamente. Hay muchos flancos por donde avanzar. Resta determinar el peso que tuvieron en la construcción material de las iglesias los diferentes niveles del Estado (nacional, provincial, e incluso municipal luego de su creación) y las distintas organizaciones de la sociedad civil en cada realidad provincial o, al menos, regional. ${ }^{46}$ También hay mucho para estudiar sobre la composición del clero argentino de mediados del siglo XIX. Cuál era su formación, su origen social, cuáles eran las concepciones eclesiológicas que orientaban sus movimientos. Es necesario, en este sentido, conocer hasta qué punto el sector ultramontano se reconocía como un grupo de intereses comunes y actuaba solidariamente para alcanzar objetivos compartidos. También debería determinarse si existían otros grupos o tendencias dentro del clero con proyectos alternativos. ${ }^{47}$

Las reflexiones desplegadas aquí pretenden ofrecer algunas premisas para orientar esas investigaciones. Así como se asume hoy que el Estado nacional no se construyó avanzando sobre las dirigencias e instituciones provinciales

\footnotetext{
46 Algo en este sentido se está haciendo para Tucumán (Ábalo, 2012, 2013).

47 Existe un estudio en curso de Roberto Di Stefano sobre el rol de la revista católica La Relijión, como órgano de expresión de este sector ultramontano, que avanza reflexiones sobre las características del grupo. Remito también a Martínez (2015).
} 
y sometiéndolas, sino que muchas veces esas dirigencias participaron de la empresa buscando ampliar sus espacios y herramientas de poder, también puede pensarse que la construcción de la Iglesia argentina bajo el signo de la romanización no fue el fruto de un avance unilateral de la Santa Sede sobre las rémoras de las iglesias coloniales rioplatenses. Dentro y fuera de las instituciones eclesiásticas argentinas existieron actores muy activos que proyectaron una Iglesia transnacional y gobernada centralizadamente desde Roma, pero participaron de esa construcción siguiendo criterios propios e imprimiendo al proceso un rumbo específico. Quizás si adquirimos esa perspectiva podamos entender mejor las particularidades del vínculo entre Estado e Iglesia en la Argentina, cuya singular persistencia aún desvela a sus estudiosos.

\section{Referencias bibliográficas}

1. Auza, N. T. (1979). La política religiosa de la Confederación. Revista Histórica, 3, 3-75.

2. Auza, N. T. (1981). Los recursos económicos de la Iglesia hasta 1853. Antecedentes del presupuesto de culto. Revista Histórica, 8, 3-28.

3. Ayrolo, V. (1996). Una nueva lectura de los informes de la misión Muzi: la Santa Sede y la Iglesia de las Provincias Unidas. Boletín del Instituto de Historia Argentina y Americana "Dr. Emilio Ravignani", 14, 31-60.

4. Ayrolo, V. (2002). Pedro Ignacio de Castro Barros (1777-1849). Publicista de Dios y de la Patria. En N. Calvo, R. Di Stefano y K. Gallo (Eds.) Los curas de la revolución. Vidas de eclesiásticos en los orígenes de la Nación (pp. 265-277). Buenos Aires: Emecé.

5. Ayrolo, V. y Mazzoni, M. L. (2013). De familiar a Obispo de Córdoba. La trayectoria política de Benito Lascano como ejemplo de ascenso en la carrera eclesiástica, 1800-1836. Anuario de la Escuela de Historia Virtual, 4, 35-56.

6. Bragoni, B. y Míguez, E. (Eds.) (2010). Un nuevo orden político: provincias y estado nacional, 1852-1880. Buenos Aires: Biblos.

7. Bruno, C. (1974). Historia de la Iglesia en Argentina, volumen XI. Buenos Aires: Don Bosco.

8. Bruno, C. (1980). Una información del Dr. Facundo Zuviría sobre eclesiásticos. (Montevideo, 6 de abril y 31 de mayo de 1856). Investigaciones y Ensayos, 28, 477-493.

9. Centeno, F. (1909). La diplomacia argentina ante la Santa Sede. Revista de Derecho, Historia y Letras, XXXII, 147-414, 467-496 y XXXIII, 44-60, 207$221,377-414$. 
10. Clark, C. M. (2003). The New Catholicism and the European Culture Wars. En C. M. Clark y W. Kaiser (Eds.) Culture Wars: Secular-Catholic Conflict in Nineteenth-Century Europe (pp. 11-46). Reino Unido-Nueva York: Cambridge University Press. DOI: 10.1017/cbo9780511496714.002 .

11. Congreso Nacional (1891). Actas de las Sesiones de la Cámara de Diputados, años 1856-1869. Buenos Aires: Compañía Sudamericana de Billetes de Banco.

12. Congreso Nacional (1891). Actas de las Sesiones de la Cámara de Senadores, años 1856-1861. Buenos Aires: Compañía Sudamericana de Billetes de Banco.

13. Di Stefano, R. y Zanatta, L. (2000). Historia de la Iglesia argentina. Desde la Conquista hasta fines del siglo XX. Buenos Aires: Grijalbo-Mondadori.

14. Di Stefano, R. (2004). El púlpito y la plaza. Clero, sociedad y política de la monarquía católica a la república rosista. Buenos Aires: Siglo XXI.

15. Di Stefano, R. (2011). Por una historia de la secularización y de la laicidad en Argentina. Quinto Sol, 15 (1). Disponible en: http://ojs.fchst.unlpam. edu.ar/ojs/index.php/quintosol/article/viewFile/116/94

16. Di Stefano, R. (2013). Sobre liberalismo y religión: rentas eclesiásticas y presupuesto de culto en el Estado de Buenos Aires (1852-1862). A/manack, 5. Disponible en: http://www.almanack.unifesp.br/index.php/ almanack/article/view/982

17. Garavaglia, J. C. (2010). Rentas, deuda pública y construcción estatal: la Confederación Argentina, 1852-1861. Desarrollo Económico, 50 (198), 223-247.

18. Lanteri, A. L. (2013a). Acerca del aprendizaje y la conformación políticoinstitucional nacional. Una relectura de la 'Confederación' argentina (1852-1862). Secuencia, 87, 69-94.

19. Lanteri, A. L. (2013b). La "Confederación" desde sus actores. La conformación de una dirigencia nacional en un nuevo orden político (1852-186). En A. L. Lanteri (Ed.) Actores e identidades en la construcción del Estado nacional (pp. 129-169). Buenos Aires: Teseo.

20. Lida, M. (2006). Una Iglesia a la medida del Estado: la formación de la Iglesia nacional en la Argentina (1853-1865). Prohistoria, 10, 27-46.

21. Lida, M. (2007). El presupuesto de culto en la Argentina y sus debates. Estado y sociedad ante el proceso de construcción de la Iglesia (1853-1880). Andes, 18, 49-75.

22. Martínez, I. (2013). Una nación para la iglesia argentina. Construcción del Estado y jurisdicciones eclesiásticas en el siglo XIX. Buenos Aires: Academia Nacional de la Historia-Dunken. 
23. Martínez, I. (2014a). Circulación de noticias e ideas ultramontanas en el Río de la Plata tras la instalación de la primera nunciatura en la América ibérica (1830-1842). Historia Crítica, 52, 73-97. DOI: 10.7440/ histcrit52.2014.04.

24. Martínez, I. (2014b). Desarrollo de la intransigencia católica en Argentina durante la primera mitad del siglo XIX. En A. C. Aguirre y E. Abalo (Eds.) Representaciones sobre historia y religiosidad. Deshaciendo fronteras (pp. 113-127). Rosario: Prohistoria.

25. Martínez, I. (2015). Consolidación del discurso ultramontano y clero intransigente en el Río de la Plata: 1820-1865. En A. Machado de Oliveira y V. Ayrolo (Coords.) Histórias de Clérigos e Religiosas nas Américas: conexões entre Argentina e Brasil (séculos XVII-XIX). Río de Janeiro: Mauad Editora (en prensa).

26. Míguez, E. (2012). Gestación, auge y crisis del orden político oligárquico en la Argentina. Balance de la historiografía reciente. Polhis, 9, 38-68.

27. Ministerio de Relaciones Exteriores de Bolivia. (1883). Datos y antecedentes para la celebración de un concordato. La Paz: La Libertad. Disponible en: http://nrs.harvard.edu/urn-3:FHCL:115945.

28. Oszlak, O. (1985). La formación del estado argentino. Buenos Aires: Editorial de Belgrano.

29. Picado G. M. (1993). Los concordatos celebrados entre los países de Centro América y la Santa Sede durante el siglo XIX. Revista de Historia (Costa Rica), 28, 207-232.

30. Registro Oficial de la República Argentina. (1882). Tomos III y IV, Buenos Aires.

31. Sábato, H. (2012). Historia de la Argentina. Buenos Aires: Siglo XXI.

32. Segura, J. J. (1964). Historia eclesiástica de Entre Ríos. Nogoyá: Imprenta Nogoyá.

33. Tonda, A. (1952). Rivadavia y Medrano: sus actuaciones en la reforma eclesiástica. Santa Fe: Castellví.

34. Tonda, A. (1956). Don Félix Frías: el secretario del general Lavalle, su etapa boliviana (1841-1843). Córdoba: Argentina-Cristiana.

35. Tonda, A. (1957). Historia del Seminario de Santa Fe. Santa Fe: Castellví.

36. Tonda, A. (1961). Castro Barros: sus ideas. Buenos Aires: Academia del Plata. 